\title{
Design and Synthesis of Visible Light Communication Transceiver on FPGA
}

\author{
A. Muhammad Sagif Shahab, B. Denny Darlis \\ Universitas Telkom \\ Bandung, Indonesia \\ sagif.shahab@telkom.co.id, denny.darlis@tass.telkomuniversity.ac.id
}

\begin{abstract}
In this paper, we designed and synthesized a Visible Light Communication (VLC) system with Inverse / Fast Fourier Transform (I/FFT) as a frequency multiplexing method using an FPGA. The system will map the data sent using a constellation mapper, which uses 4-Quadrature Amplitude Modulation. After mapped, the data is frequency multiplexed using an IFFT block and converted into its analog form before being transmitted through an LED amplifier circuit and a Cree XML2 LED. Next, the signal is received by Ba PV10 photodiode on the receiver module and a transimpedance amplifier. Once processed by the VLC receiver, the conversion of analog signals to digital is done before demultiplex the signal using FFT. Then we demapped the signal from back to the original data using a 4QAM demapper. The transceiver system was implemented on Altera De-0 Nano board with an Altera Cyclone 4E FPGA. The VLC transmitter module, VLC receiver, and ADC / DAC were implemented on a breadboard. The communication system works with a bit rate of $302 \mathrm{kbps}$. The Analog and digital subsystems are capable of supporting the bitrate, and supplying the LED lamp with a power of $0.10125 \mathrm{~W}$. The system was then tested at different distances from $25 \mathrm{~cm}$ to $60 \mathrm{~cm}$. At 25 and $30 \mathrm{~cm}$, there is no error on the characters received. But at $35 \mathrm{~cm}$ there was a character error rate of $0.042 \%$. This error continued to increase as the distance was added so that it reached $7.42 \%$ at a distance of $60 \mathrm{~cm}$. This is normal since, at a distance of 25 and $30 \mathrm{~cm}$, the signal received was still large so that the SNR was still good. But at $35 \mathrm{~cm}$ onwards, the received signal started to distort and the SNR started to decrease.
\end{abstract}

Index Term-Digital System; FPGA; Inverse / Fast Fourier Transform; Visible light communication.

\section{INTRODUCTION}

Visible Light Communication (VLC), can be a very good alternative for data communications. This is due to LED technology that has continued to emerge since the first time discovered in 1962[1]. Currently, LEDs are getting brighter so it is capable of being used as a lighting device, so the LEDs slowly start batting the ordinary light bulbs [7][8]. This raises an opportunity to integrate visible light communications to existing lighting such as at home, office, etc. Meanwhile Fast Fourier Transform has become a very wide algorithm used in the broadband wireless telecommunication system, especially on multicarrier transmission systems.

This paper discusses the implementation of a visible light communication system with I/FFT as Frequency multiplexing method / demultiplexing frequencies using FPGA. In FPGA the digital system consists of two blocks, the transmitter and the receiver. The data was provided by any kind of $\mathrm{PC}$ via a USB-to-serial converter and received by the FPGA transmitter. Then we mapped the data into constellation mapping using 4QAM and multiplexed frequency with IFFT. After that, the resulting signal is converted to an analog signal before it is transmitted with LED and amplifier circuit on VLC transmitter. The signal was then received by a photodiode and a transimpedance amplifier on the VLC receiver. Once received, the analog signal was converted back into a digital signal, and a frequency demultiplexing was performed using FFT in FPGA system at the receiver part. Finally, the system performed demapping from the constellation into the original data using 4QAM so it translated back to original data.

\section{BASIC THEORY AND DESIGN}

\section{A. Fast Fourier Transform}

Fast Fourier Transform (FFT) is an algorithm for calculating the Discrete Fourier Transform (DFT) of a signal quickly [2]. Just like DFT, FFT changes the signal from time domain to domain frequency. But FFT is much more efficient than DFT because it requires a simpler circuit and fewer mathematical operations. Inverse Fast Fourier Transform performs the opposite of FFT, ie converting a frequency domain signal into a time domain. The FFT block used in this study uses the FFT block synthesized in the study earlier [3]. This FFT block has the specifications as described on Table I.

Since FFT describes the relationship between the time spectrum and the frequency spectrum, the FFT is used for frequency multiplexing [4]. Frequency multiplexing is basically combining several signals that have different frequencies into one new signal. If we modify the frequency spectrum of the signal, we indirectly modify the constituent signals. Modifications on the frequency spectrum are what can be done by FFT so it can be said FFT is capable of doing frequency multiplexing. It is this capability that enables multicarrier transmission using FFT. Generally, the transmitter uses Inverse FFT and the receiver uses FFT.

Table I Specification of the FFT block used in this paper

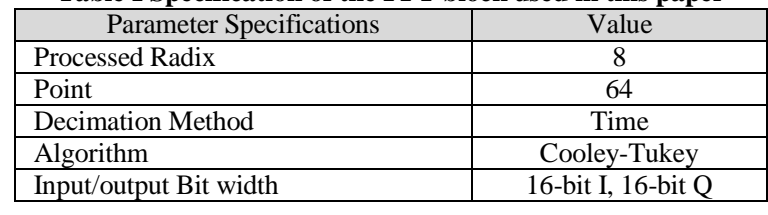




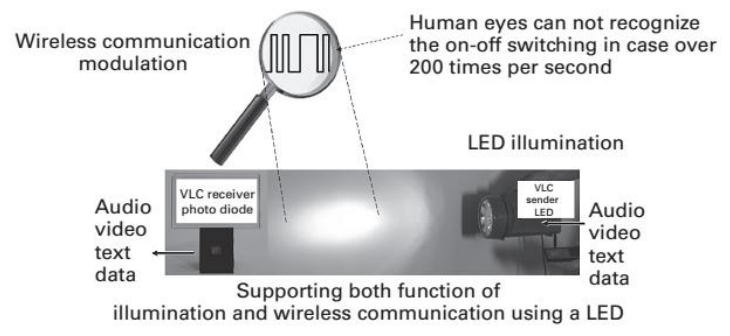

Figure 1 Typical system for Visible Light Communication [1]

\section{B. Visible Light Communication}

VLC is one method of data communication that uses visible light as its signal. The visible light used is the illumination lamp. Visible light can be made from a single LED or multiple LEDs. VLC transmitter blocks are generally implemented with LEDs or light bulbs. Electrical signals are generated by a source (computer, cell phone etc) forwarded to an LED driver. The drivers supports LEDs enough current to illuminate. After that the signal is converted to light by the LED and received by the block receiver. The receiver block is implemented with a photodiode that converts the light signal back to electrical signals [9]. This electrical signal is still a current so it must be converted into a voltage signal with a transimpedance amplifier[5][6][10]. The visible light communication typical system is generally shown in Figure 1.

\section{SySTEM DESIGN AND IMPLEMENTATION}

Overall the system has digital parts and analog parts. The digital part is implemented using FPGA, while the analog part is implemented with discrete components. The digital system in the FPGA is implemented into the transmitter and the receiver block. The overall implementation is shown by the block diagram in Figure 2.

A more complete diagram of the FPGA digital transmitter and receiver design is shown in Figure 3. From the figure, it is shown that both FPGA systems needs additional blocks such as RAM, ADC Controller and DAC Controller. The ADC Controller function is to control Analog-to-Digital chip connected to FPGA. DAC Controller block will control the Digital-to-Analog chip. The RAM holds temporary data before and after conversion by I/FFT. The synchronization block was not implemented since the data transmission was assumed to be synchronous.

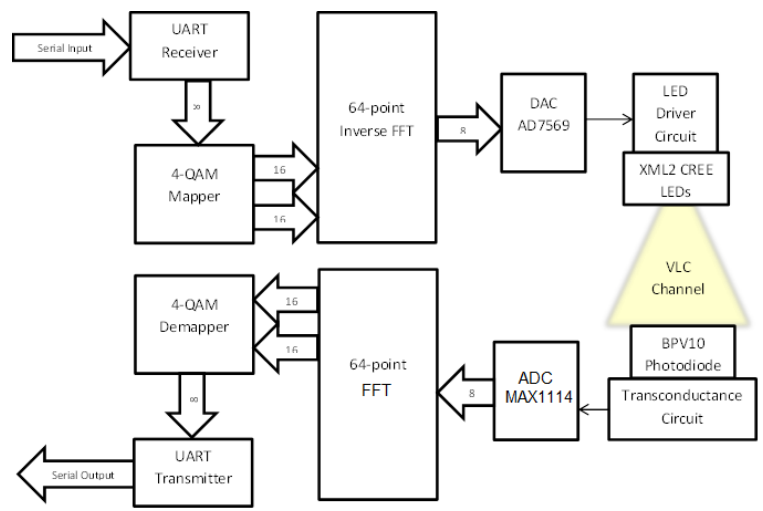

Figure 2 Communication System Block Diagram
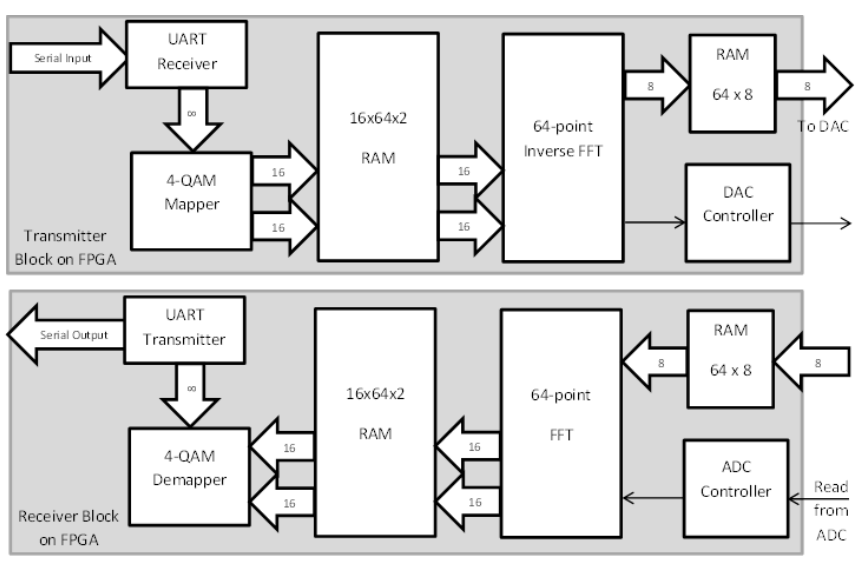

Figure 3 More complete diagram blocks of the Transmitter and Receiver designed on FPGA

As the system is assumed to be synchronous, there is a dummy sync signal between the transmitter and the receiver blocks whose function is to acknowledge the receiver whether the transmission process is being started.

The function of the IFFT block is to do frequency multiplexing, whereas the FFT block does the opposite, frequency demultiplexing. Number of subcarriers used in this multiplexing frequency process there are 28 of 64 subcarriers. The first subcarrier is DC, while subcarriers 29, 30 and 31 weren't used. The remaining 32 subcarriers are Hermitian Symmetry mirrors, for IFFT output to be real.

\section{A. Implementation of Digital System In FPGA}

We designed the digital system in hierarchical level, so that the functionality would be appropriate with predicted results. The hierarchical structure of the designed block is shown in Figure 4.

After we verify the timing simulation, then the design blocks were synthesized using Quartus II software from Altera and generate the RTL schematic as shown in Figure 5. This schematic shows each block that has been designed and tested by the simulation. The digital transmitter and receiver are combined into one VHDL file "ofdm_modem.vhd".

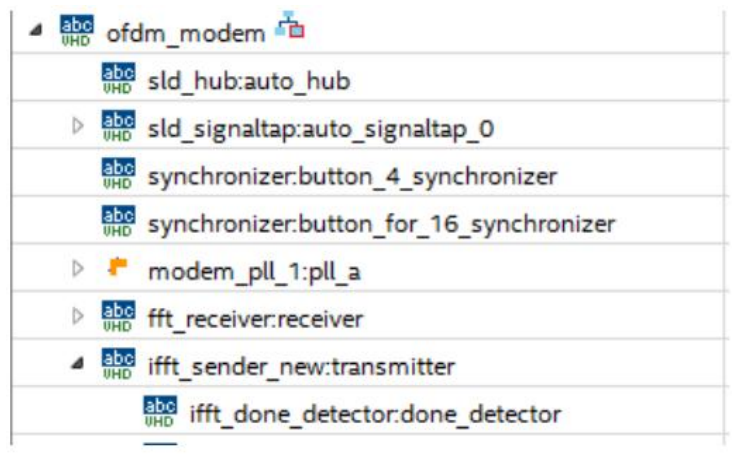

Figure 4 Hierarchy structure for "ofdm_modem.vhd" for VLC 


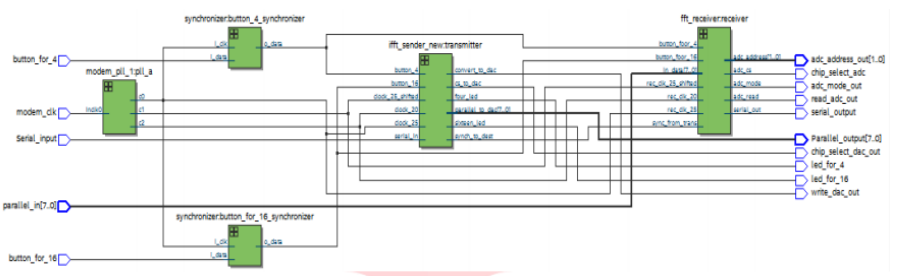

Figure 5 RTL Schematic circuit The top hierarchy "ofdm_modem.vhd" after synthesis

Synthesis is the process of converting VHDL code into RTL circuit. The result of synthesis is information on FPGA resource utilization at the RTL level shown in Table II.

After the synthesis was done, the next process was placing pin location (pin placement). In the process, the pins in the top hierarchical VHDL module were mapped to physical pins on the FPGA. Then, a place-and-route process was performed. In this process, the RTL circuit of synthesis was converted into a physical circuit in FPGA along with its placement. In the place-and-route process, the placement method was based on the desired timing specification to obtain the FPGA floorplan. The resulting resource utilization was changed a bit because of the obligation to meet the timing specification, therefore the software modified the resource utilization to fulfill that obligation. Then after the place-and-route process, a timing analysis was performed to obtain the maximum operating clock speed of the circuit. In this system, we used an Altera Cyclone 4E low-cost development board with a default clock of $50 \mathrm{MHz}$. A digital phase locked loop (PLL) was synthesized on-chip to convert this clock into three clock signals.

Table II FPGA element utilization for this paper

\begin{tabular}{|l|c|}
\hline \multicolumn{1}{|c|}{ Resource Utilization } & Value \\
\hline Logic Elements & 9707 \\
\hline Registers & 3047 \\
\hline Pin Utilization & 30 \\
\hline Memory Used (bit) & 13440 \\
\hline Multplier 9-bit Used & 48 \\
\hline PLL & 1 \\
\hline
\end{tabular}

After that, the completed digital system was ready to be programmed into FPGA. In this research, the programming file format used was the .jic format. The .jic format is a format for programming flash on FPGA boards by using JTAG. This .jic format was obtained after conversion from the .sof format. This non-volatile format was used so that when FPGA was switched off, the program wasn't deleted.

\section{TESTING AND VERIFICATION}

The test was conducted over various distances from $30 \mathrm{~cm}$ to $60 \mathrm{~cm}$, with each distance tested three times. An example of the signal conditions before and after being transmitted is displayed by the oscilloscope as shown in Figure 6, for the distance of $30 \mathrm{~cm}$.

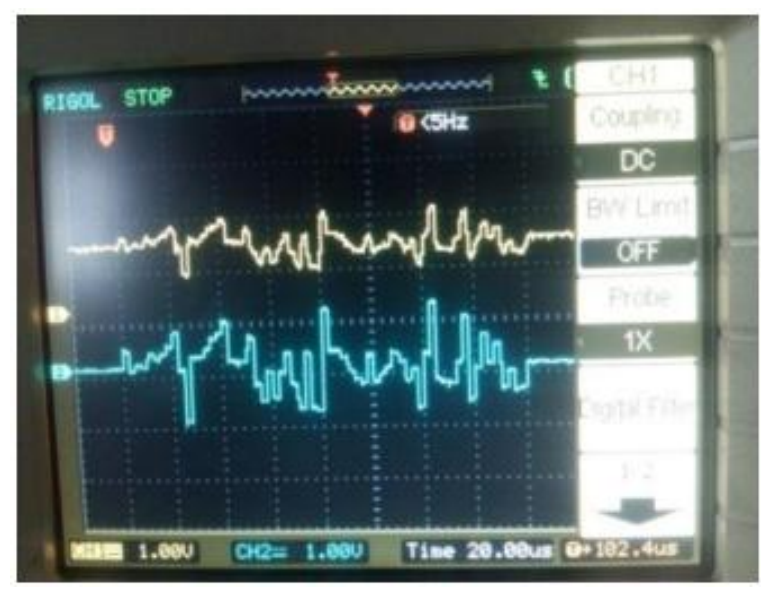

Figure 6 Transmitted (blue) and Received (yellow) signal at $30 \mathrm{~cm}$ distance for "mother" ASCII characters

At a distance of $30 \mathrm{~cm}$ and below, there was no error because the received signal was still large enough and the SNR was still good. Above $30 \mathrm{~cm}$, the received signal began to weaken and character errors started to appear as shown in Table III and Figure 7.

Table III Character error rate after the experiment was conducted

\begin{tabular}{|c|c|c|c|c|}
\hline $\begin{array}{c}\text { Distance } \\
(\mathrm{cm})\end{array}$ & $\begin{array}{c}1 \mathrm{st} \\
\text { experiment }\end{array}$ & $\begin{array}{c}\text { 2nd } \\
\text { experiment }\end{array}$ & $\begin{array}{c}\text { 3rd } \\
\text { experiment }\end{array}$ & $\begin{array}{c}\text { Average } \\
\text { error }\end{array}$ \\
\hline 30 & $0 \%$ & $0 \%$ & $0 \%$ & $0 \%$ \\
\hline 35 & $0,050 \%$ & $0,025 \%$ & $0,050 \%$ & $0,042 \%$ \\
\hline 40 & $0,220 \%$ & $0,320 \%$ & $0,200 \%$ & $0,247 \%$ \\
\hline 45 & $0,770 \%$ & $0,830 \%$ & $0,650 \%$ & $0,750 \%$ \\
\hline 50 & $2,390 \%$ & $2,630 \%$ & $2,480 \%$ & $2,470 \%$ \\
\hline 60 & $7,470 \%$ & $7,360 \%$ & $7,440 \%$ & $7,420 \%$ \\
\hline
\end{tabular}

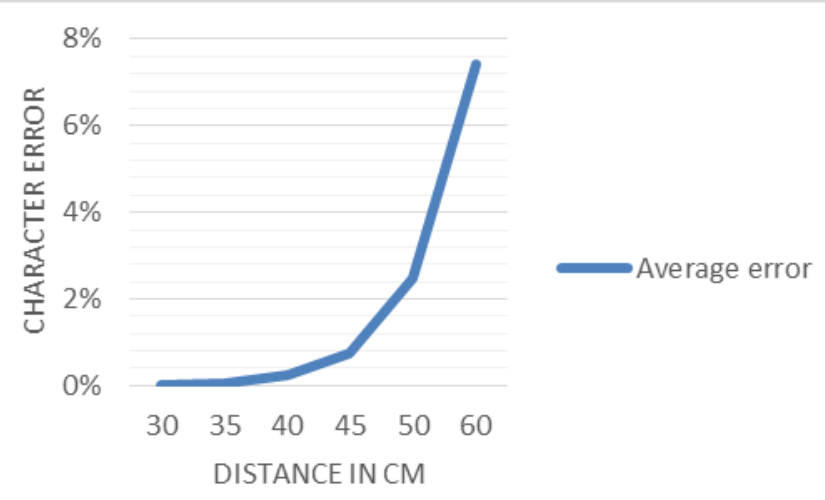

Figure 7 Average character error percentage over various distances

\section{Conclusions}

In this paper, the following conclusions are obtained:

1. FPGA Transmitter and FPGA Receiver in this research can be applied into the Altera Cyclone 4E FPGA contained in the Altera DE0-Nano board with using the resource as optimal as possible. 
2. ADC and DAC subsystems have sufficient sampling speed, ie $400 \mathrm{ksps}$ so the system can reach a bitrate $302 \mathrm{kbps}$

3. The VLC transmitter subsystem and VLC receiving subsystem can be tested at distances up to $60 \mathrm{~cm}$ with minimum distortion so that the bandwidth of both subsystems is sufficient for this research. The main constraint is in the LED emitting power so that at a distance of $60 \mathrm{~cm}$ the signal looks small.

4. The system transmits error-free data up to a distance of $30 \mathrm{~cm}$. At a distance of $35 \mathrm{~cm}$, character errors started appearing at an average rate of $0.042 \%$. At a distance of $60 \mathrm{~cm}$, this average error rate reached $7.42 \%$. At the distance of $30 \mathrm{~cm}$, the signal power was still large so the SNR was still good. At distances of $35 \mathrm{~cm}$ and above, the signal power began to decrease so that SNR was distorted. The further the distance, the weaker the received signal. This weaker signal led to lower SNR which in turn led to greater character error rate.

\section{ACKNOWLEDGMENT}

The authors would like to acknowledge the invaluable contributions in Universitas Telkom, Bandung to many of the results and work described here.

\section{REFERENCES}

[1] S. Arnon,"Visible Light Communication", Cambridge University Press, 2015.

[2] J. Ifeachor. Digital Signal Processing, A Practical Approach. Harlow, The United Kingdom: Prentice Hall, 2002.

[3] D. Darlis, A.A.Muayyadi, S. Sumaryo,"Perancangan dan Implementasi Prosesor OFDM Baseband Untuk Prototipe Modem PLC Pada FPGA," Jurnal Penelitian dan Pengembangan Telekomunikasi, Volume 15, No. 2 , 2010

[4] M. J. F. Manalu, I. Hidayat, D. Darlis, “ Desain dan Implementasi Prosesor I/FFT 512 Titik Radix-8 pada FPGA untuk Broadband Wireless Access", Jurnal Tektrika, Vol. 2. No.1, In-Press, 2017

[5] National Taiwan University.”Orthogonal Frequency Divison Modulation",

https://www.csie.ntu.edu.tw/ hsinmu/courses/_media/wn_11fall/ofdm_n ne.pdf [Accessed 5 Juni 2016]

[6] R.C. Jaeger, T.N. Blalock, "Microelectronic Circuit Design”, Fourth Edition. The United States: McGraw-Hill, 2011.

[7] B. Pease. Electronics Design Website. "What's All This Transimpedance Amplifier Stuff, Anyhow? ", http://electronicdesign.com/analog/whats-all-transimpedance-amplifierstuff-anyhow-part-1 [Accessed 21 Oktober 2015]

[8] Edison Tech Center. "LEDS and OLEDS", http://www.edisontechcenter.org/LED.html [ Accessed 1 November 2015]

[9] J. Layton. "How LED Light Bulbs Work", http://science.howstuffworks.com/environmental/greentech/sustainable/led-light-bulb2.htm [Accessed 10 February 2016]

[10] I. Poole, "Radio Electronics - PIN/PN Photodiode", http://www.radioelectronics.com/info/data/semicond/photo_diode/pin-pn-photodiode.php [Accessed 10 February 2016] 